\title{
Mitigating signaling congestion in LTE location management by overlapping tracking area lists
}

\author{
Sara Modarres Razavi and Di Yuan
}

\section{Linköping University Post Print}

N.B.: When citing this work, cite the original article.

Original Publication:

Sara Modarres Razavi and Di Yuan, Mitigating signaling congestion in LTE location management by overlapping tracking area lists, 2012, Computer Communications, (35), 18, 2227-2235.

http://dx.doi.org/10.1016/j.comcom.2012.08.008

Copyright: Elsevier

http://www.elsevier.com/

Postprint available at: Linköping University Electronic Press

http://urn.kb.se/resolve?urn=urn:nbn:se:liu:diva-86384 


\title{
Mitigating Signaling Congestion in LTE Location Management by Overlapping Tracking Area Lists
}

\author{
Sara Modarres Razavia,*, Di Yuan ${ }^{\mathrm{a}, \mathrm{b}}$ \\ ${ }^{a}$ Department of Science and Technology, Linköping University, Sweden \\ ${ }^{b}$ Ericsson Research, Ericsson AB, Sweden
}

\begin{abstract}
Avoiding signaling congestion is a key aspect in location management of cellular networks. The signaling load in this context corresponds to the use of location update and paging messages for tacking user equipments (UEs). Signaling congestion may occur due to many UEs behaving in a similar manner, e.g., massive and simultaneous UE mobility in a train movement scenario. For Long Term Evolution (LTE) networks, location management can be based on the use of tracking area lists (TALs), each being a list containing multiple tracking areas (TAs). In this paper, the scheme of using TALs with overlapping TAs is applied for signaling congestion mitigation. One or multiple TALs that potentially overlap are allocated to each cell, and the TALs are assigned to the UEs that make their location updates in the cell. We derive linear programming formulations for finding the optimal proportional use of overlapping TALs for congestion avoidance. The main advantage of our approach is that it does not require the collection of detailed UE mobility information apart from what is available to the network. We report numerical results for realistic scenarios. The optimization approach yields
\end{abstract}

\footnotetext{
${ }^{*}$ Corresponding author.
} 
promising results for reducing signaling congestion, and the performance region of optimized overlapping TALs goes significantly beyond the capability of the conventional TA scheme.

Keywords: location management, long term evolution, signaling congestion mitigation, tracking area list.

\section{Introduction}

Location management is of fundamental importance in cellular communications. In Long Term Evolution (LTE) networks, the tasks are performed by the Mobility Management Entity (MME). The basic concept for tracking user equipments (UEs) is area partitioning. The network is divided into tracking areas (TAs), each consisting of a subset of the cells [1]. Typically, most network-registered UEs are in idle mode, and form the bulk of location management in the network. The network has the knowledge of the location of LTE-idle UEs to the granularity of TA. The MME location register connects each UE with its currently located TA. In the conventional TA scheme, a UE performs a TA update (TAU) when moving across its current TA boundary, by sending an uplink signaling message to the MME. When a call has to be placed to a UE, the MME sends downlink paging signaling messages to the cells inside the UE's current TA, in order to learn the specific cell to which the call should be directed. Although the term TA is LTE-specific, this basic location management scheme is adopted also in other network types, in form of the Location Area (LA) concept in the circuit-switched domain of GSM, and the Routing Area (RA) concept in the packet-switched domain of GPRS and UMTS networks. 
In designing the layout of TAs, signaling overheads generated by TAU and paging messages pose a major concern, leading to two types of optimization problems. The first is to minimize the sum (or equivalently the average) of TAU and paging overheads in the network. The second takes a load balancing perspective, with the performance target of reducing congestion, i.e., to avoid heavy amount of signaling overhead in any part of the network caused by many UEs behaving in a similar manner, e.g., massive and simultaneous UE mobility in a train scenario.

The rationale of avoiding signaling congestion is to ensure no significant degradation in the quality of service, which may occur due to resource exhaustion for tracking UEs. In addition to the train scenario, there are several types of mobility patterns that may cause TAU congestion. For example, in densely-populated cities, there is a drastic difference in the mobility between day and night. That a very large number of UEs moving concurrently into a central area (typically early morning of a weekday) may generate excessive signaling around the center $[3,13,14]$. Conversely, heavy paging may occur as a result of massive and close-to-static UEs simultaneously located at some hotspot (e.g., a large stadium).

In this paper, we present an optimization framework for mitigating signaling congestion, based on the use of LTE tracking area lists (TALs) that may contain overlapping TAs. TAL is an LTE-specific concept for location management [2]. Instead of belonging to one TA, a UE can be assigned a list of TAs, referred to as a tracking area list. The UE makes a TAU when it moves into a TA that is not part of the list. Thus, the UE's location is known to the MME to at least the accuracy of the list allocated to that UE. 
Moreover, a cell can assign different TALs to its UEs, and the TALs used by the network may overlap in their TA elements. As the creation and reconfiguration of TALs are supported in the LTE network architecture, the optimization framework we propose is cost-efficient in implementation.

The optimization framework for overlapping TALs can be used for reducing TAU or paging signaling congestion, depending on network configuration and scenario (massive mobility or UE gathering). The flexibility in forming TAs is related to the sizes of its building elements (i.e., TAs). The most flexible setting is that each cell (or site) is considered a TA, and the effect of larger TAs is implemented by TALs $[8,16,17]$. For reducing TAU, this setting is not necessary, as overlapping TALs can be created and applied to any given TA layout in order to yield a "blurring effect" of the TA boundaries, with the effect of smearing the TAUs over a larger area. For reducing paging congestion, TALs are assumed to be in place in form of lists of site-based TAs. For areas with risk of paging signaling congestion, new and overlapping TALs can be created inside the original ones. We will deal with both cases in this paper, with focus on TAU congestion due to massive mobility, assuming that paging will be a less critical issue in real-life deployments.

A major difficulty in optimizing TALs is to assess its performance in the planning stage. For conventional TAs, both the TAU and paging overhead can be effectively assessed, because the boundaries between TAs are sharp, and all UEs in the same location are consistent in whether or not TAU is required, which is indeed the cause of potential TAU congestion in case of massive mobility. In the literature (e.g., [27]), handover and cell-load statistics of active UEs, that also represent accurately the behavior of the idle ones, 
are commonly used as input for optimal TA design. For TALs, UEs at the same location may in general hold different lists, and thus where TAUs will be made depend on the specific mobility pattern of each individual UE. This information is however not available. Thus, it is hard to calculate or estimate the performance of candidate TAL configurations in advance. One approach for circumventing the issue is to use mobility models [8, 16, 17]. However, the performance is highly sensitive to the validity of the assumptions made in the models.

Our optimization framework for overlapping TALs overcomes the uncertainty in modeling UE mobility, as no mobility model or detailed UE movement information is required. Yet, performance evaluation is straightforward because, by construction, all sites in the same area will assign TALs consistently based on their proportional usage. Computational results show that the optimization approach is highly promising for mitigating signaling congestion, and optimized overlapping TALs are able to perform significantly better than what ultimately is achievable by the conventional TA scheme.

The remainder of the paper is organized as follows. In Section 2 we review some works that are relevant to our study. Section 3 is devoted to basic notation and an illustrative example of the optimization approach. We detail the optimization formulations using the overlapping TAL scheme for congestion avoidance of TAU and paging in Sections 4 and 5, respectively. In Section 6, we present results of performance evaluation of the proposed approach and compare it to the conventional TA scheme. Conclusions are provided in Section 7. 


\section{Related Works}

For surveys of performance engineering in location management, we refer to $[5,30]$. Among the engineering tasks, planning and optimization of TAs (or LAs and RAs for previous generations of cellular networks) considering signaling overhead have been dealt with extensively. For models and algorithms of TA layout optimization and re-configuration, we refer to $[6,7,9,15,27,28]$ and the references therein. Another line of research is the design of unconventional location update and paging schemes, such as mobility-model based TAU and sequential paging, see, for example, [4, 21, 22, 29]. Signaling congestion avoidance and load balancing inside the location management system are investigated in $[13,23,26,31]$. In [13], randomized delay in cell re-selection of UEs is used to smooth out the signaling peak generated by massive mobility. In [31], the authors present a queuing model for location updates by group mobility, and examine the performance of using an information buffer at base stations to reduce update failures. Distributed database solutions for location management are proposed in $[23,26]$. The main contributions of $[23,26]$ are models and algorithms for load balancing among the database servers in location update. Most of the mechanisms of unconventional update and paging schemes however require the use of non-standard system elements and parameters.

Recently, there has been an increasing amount of attention on the development of using the concept of TALs for location management in LTE $[14,20,24,25,32]$ in order to provide better flexibility over the conventional TA concept. In the conventional case, TAs are disjoint, and each cell is in exactly one TA. Two schemes have been considered for extending the TA 
to lists of TAs. The first scheme is to allow a cell to simultaneously belong to multiple TAs (in form of a list), where each UE registered by the cell is assigned one of the TAs from the cell's list. In the second scheme, every cell belongs to only one TA, but a UE can be assigned a list of TAs, with the effect that TAU takes place only if the UE moves outside the union of the TAs in the assigned list. The assignment of TAL may differ by UE. Both schemes are mainly intended to tackle issues related to TAU, assuming that the problem of paging is less critical. The optimization framework in this paper can be adapted to both schemes. Merely for unifying the presentation and easing the comparison with the conventional TA concept, from now on and throughout the rest of the paper, we will adopt the latter scheme, that is, TAL is considered from a UE perspective.

TAL is promising for solving some issues imposed by the conventional TA scheme. For example, TAL can be used to prevent the frequent updates when a UE keeps hopping between two or more adjacent cells in different TAs (aka the ping-pong effect) [20]. Another example is the aforementioned train scenario associated with high uplink signaling traffic due to simultaneous updates of massive UEs crossing a TA boundary along a line [14, 18]. The current paper provides significant extensions of [18] in two aspects. First, the TAL optimization framework is generalized from a line scenario (modeling train movement) to arbitrary network topology. Second, we show how the optimization approach can be adapted to downlink signaling for reducing peak paging overhead.

TAL provides a high level of flexibility. In fact, if a UE's location and mobility pattern were completely known, then the network can tailor a UE- 
specific TAL that virtually eliminates tracking signaling for the UE. In reality, however, the exact UE's future trajectories are not available, and models and algorithms for TAL configuration at a more aggregate level are required.

Previous studies of using TAL for minimizing the overall signaling overhead, with the assumption that a cell gives one common TAL to all UEs getting updated in the cell, have been presented in $[8,16,17]$. TALs, in forms of rings of cells, are used in [8]. In $[16,17]$, each cell has a cell-specific TAL to be assigned to all users registered or updated in the cell. To optimize the composition and use of TALs, one has to take into account the fact that, in general, UEs in the same location may hold different TALs, in contrast to the conventional TA case. This heterogeneity impacts on the signaling overhead. Clearly, imposing assumptions on UE behavior, ranging from simple rules of thumb to statistical mobility models of UE trajectory, constrains the applicability of TAL optimization. Nevertheless, previous works, in particular $[16,17]$, show TAL is promising in extending the capability of the conventional TA concept.

Our application focus is signaling congestion mitigation. The TAL scheme proposed in the current paper allows a cell to have a collection of potentially overlapping TALs to be assigned to its UEs. The cells are fully consistent in the proportional use of the correlated TALs. As a result, signaling overhead estimation becomes straightforward, and no assumption of UE trajectories is required. Moreover, the TAL scheme we present is coherent with the LTE system architecture, and thus it is cost-efficient to implement. 


\section{System Model: Basic Elements}

Denote by $\mathcal{N}=\{1, \ldots, N\}$ the set of sites in an LTE network. In reallife networks, a site may consist of several cells. Splitting the cells of a site into different TAs is however not a common practice. Therefore, throughout the remainder of the paper, TA and TAL will be defined on the site level. This does not impose any loss of generality, as our framework generalizes straightforwardly to the cell level. A TA design is a partitioning of the network into groups of sites. The design can be represented by an $N \times N$ symmetric and binary matrix $\boldsymbol{S}$, in which the entry at row $i$ and column $j$, denoted by $s_{i j}$, indicates whether or not sites $i$ and $j$ are in the same TA, i.e.,

$$
s_{i j}= \begin{cases}1 & \text { if sites } i \text { and } j \text { are in the same TA } \\ 0 & \text { otherwise. }\end{cases}
$$

TAs, as well as LAs and RAs in other cellular network types, do not overlap. As a result, the $\boldsymbol{S}$-matrix has the transitive property, meaning that for any three sites $i, j$, and $k, s_{i j}=s_{j k}=1$ implies $s_{i k}=1$.

Two types of input data, representing the UE location and mobility behavior for a given time period of interest, are used for performance evaluation of a TA design. Denote by $u_{i}$ the total number of UEs of site $i$, scaled by the time proportion that each UE spends in site $i$. For UE movement, denote by $h_{i j}$ the number of UEs having handover from site $i$ to site $j$. Parameters $u_{i}$ and $h_{i j}$ can be effectively obtained by site load and handover statistics of active UEs [15]. It is very reasonable to assume that the behavior of idle UEs is close or identical to that of the active UEs, hence the cell load and handover 
statistics can be used for performance evaluation of the signaling overhead. The amount of overhead of one TA update and one paging are denoted by $c^{u}$ and $c^{p}$, respectively. The exact relationship between $c^{u}$ and $c^{p}$ depends on radio system specification [10]. We use parameter $\alpha$ to denote the call intensity factor (i.e., the probability that a UE has to be paged within the time period).

Denote by $C^{U}$ the total TAU signaling overhead for the given TA design $\boldsymbol{S}$. UE movements between two sites $i$ and $j$ contribute to $C^{U}$ if and only if the two sites are in different TAs, leading to the following equation.

$$
C^{U}=\sum_{i \in \mathcal{N}} \sum_{j \in \mathcal{N}: j \neq i} c^{u} h_{i j}\left(1-s_{i j}\right)
$$

For paging a UE, a message is sent to all sites of the TA in which the UE is currently registered. Paging overhead is generated in each of the sites in the TA, except the site giving the positive response, i.e., the site where the UE is actually located. Thus, the overall paging overhead is given by (3), in which the total paging overhead generated by site $i$ to other sites in the same TA is determined by the load of site $i$ and the TA size. Note that inner sum is zero, if site $i$ forms its own TA.

$$
C^{P}=\sum_{i \in \mathcal{N}} \sum_{j \in \mathcal{N}: j \neq i} \alpha c^{p} u_{i} s_{i j}
$$

From Equations (2) and (3), it is seen that TA design has to deal with a trade-off between $C^{U}$ and $C^{P}$. Having very small TAs (e.g., one site per TA) virtually eliminates paging, but causes excessive TAU, whereas TAs of too large size give the opposite effect. 


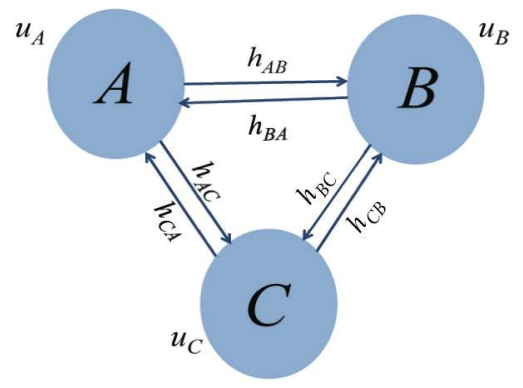

Figure 1: A small example with three sites.

Table 1: Possible TA configurations of the network in Figure 1.

\begin{tabular}{lll}
\hline \multicolumn{1}{c}{ Configuration } & \multicolumn{1}{c}{$C^{U}$} & \multicolumn{1}{c}{$C^{P}$} \\
\hline 1. $\{A\},\{B\},\{C\}$ & $c^{u}\left(h_{A B}+h_{B A}+h_{A C}+h_{C A}+h_{B C}+h_{C B}\right)$ & 0 \\
\hline 2. $\{A, B\},\{C\}$ & $c^{u}\left(h_{A C}+h_{C A}+h_{B C}+h_{C B}\right)$ & $\alpha c^{p}\left(u_{A}+u_{B}\right)$ \\
\hline 3. $\{A, C\},\{B\}$ & $c^{u}\left(h_{A B}+h_{B A}+h_{B C}+h_{C B}\right)$ & $\alpha c^{p}\left(u_{A}+u_{C}\right)$ \\
\hline 4. $\{A\},\{B, C\}$ & $c^{u}\left(h_{A B}+h_{B A}+h_{A C}+h_{C A}\right)$ & $\alpha c^{p}\left(u_{B}+u_{C}\right)$ \\
\hline $5 .\{A, B, C\}$ & 0 & $\alpha c^{p}\left(u_{A}+u_{B}+u_{C}\right)$ \\
\hline
\end{tabular}

We illustrate the trade-off and introduce the TAL scheme using a small example of three sites $A, B$, and $C$, see Figure 1. Here, the term "site" shall not be interpreted in a restrictive sense, as the example is easily generalized to the case where the three elements $A, B$, and $C$ in the figure are TAs, each containing multiple sites. With the conventional TA concept, there are in total five possible design solutions, as summarized in Table 1. The table also display the corresponding TAU and paging overheads for these configurations, calculated according to Equations (2) and (3).

Consider the total TAU overhead (i.e., $C^{U}$ ). The value is either zero, or a sum taken over some pairs of sites (e.g., $c^{u}\left(h_{A B}+h_{B A}\right)$ for sites $A$ and $B$ ); no other values are attainable. This also holds in general, because the TA 
boundaries are sharp by the conventional TA scheme.

Suppose $A, B$, and $C$ are (single-sited) TAs, and TALs which may partially overlap in their compositions are created and assigned to UEs. There are a total of seven possible TALs, each being a non-empty subset of $\{A, B, C\}$ for the example in Figure 1. Any UE in this network will hold one of the seven TALs. Note that the three network elements have their respective applicable collections of TALs. For each site, the collection is formed by the TALs containing the site. For example, $A$ will potentially assign four TALs to UEs registered or updated in $A:\{A\},\{A, B\},\{A, C\},\{A, B, C\}$. Each of the four TALs has a proportional value of usage by $A$, and the values sum up to one. Clearly, among UEs that are updated by $A$, the percentage of UEs holding each of the four TALs will follow the proportional value used by $A$ for the TAL.

Consider, for example, TAL $\{A, B\}$. The TAL appears in the collections of both $A$ and $B$. If the two sites apply different proportional values to this TAL, then the overall proportion of UEs holding the TAL in the area defined by $\{A, B\}$, including those performing handover in the two directions, will depend on the UE mobility behavior. In this case, it becomes very hard to calculate the number of UEs holding $\{A, B\}$ without imposing assumption on the mobility pattern. To tackle this difficulty, in our optimization framework, it is required that $A$ and $B$ apply the same proportional value for the TALs containing both $A$ and $B$. This is generalized to any TAL, that is, all sites within a TAL are fully consistent in their proportional use of the TAL. Denote by $x_{\ell}$ a non-negative optimization variable representing the proportional usage of TAL $\ell$ by all sites (or, TAs in general) within list $\ell$. The 
solution space of applying overlapping TALs to the three-elements network is given by the equations below. For convenience, we use the composition of $\ell$ as subscript, and skip any explicit set notation for TAL in the three equations.

$$
\begin{aligned}
& x_{A}+x_{A B}+x_{A C}+x_{A B C}=1 \\
& x_{B}+x_{A B}+x_{B C}+x_{A B C}=1 \\
& x_{C}+x_{A C}+x_{B C}+x_{A B C}=1
\end{aligned}
$$

Because the usage of any TAL $\ell$ is unified within the entire service area of $\ell$, no matter if the UEs are currently stationary or making handover between sites in $\ell, x_{\ell}$ accurately provides the proportion of UEs holding $\ell$ among all UEs in the area. Also, note that these UEs could have received list $\ell$ from any of the sites in the list.

The TAU and paging overhead of the proposed TAL scheme are determined by the $x$-variables, and the computation does not require any additional data apart from what already is available in form of cell load and handover statistics. For example, consider the amount of TAUs between $A$ and $B$. The number of UEs performing handover in the direction $A \rightarrow B$ is represented by the handover statistic $h_{A B}$. Among them, a UE makes a TAU if and only if its TAL does not contain $B$. Therefore, the TAU is $c^{u} h_{A B}\left(x_{A}+x_{A C}\right)$. For the opposite direction, the value is $c^{u} h_{B A}\left(x_{B}+x_{B C}\right)$. Recall that, using the conventional TA concept, the total amount of TAU signaling overhead between the two sites is either zero, or $c^{u}\left(h_{A B}+h_{B A}\right)$. With the overlapping TAL scheme, the TAU becomes $c^{u} h_{A B}\left(x_{A}+x_{A C}\right)+c^{u} h_{B A}\left(x_{B}+x_{B C}\right)$. As 
$x$-variables are allowed to be fractional, the amount of TAU signaling may attain values in the range $\left[0, c^{u}\left(h_{A B}+h_{B A}\right)\right]$. This observation intuitively provides the potential of the proposed TAL scheme for congestion mitigation. In other words, whereas any conventional TA design $\boldsymbol{S}$ is constrained to satisfy transitivity, TAL does not have this limitation.

\section{TAL Optimization for Congestion Mitigation of TAU}

In this section, we generalize the previous example and formalize the optimization approach for TAU congestion mitigation, e.g., the train scenario, by optimizing the TALs for a network region where massive TAU is of concern. All sites in one TA will use the same collection of TALs, and they update their UEs with the same proportional use of each TAL. We consider a conventional TA design as the underlying structure. For the network region in question, denote the current TAs forming the region by set $\mathcal{T}=\{1, \ldots, T\}$. Each TA $v \in \mathcal{T}$ is a set of sites. The TAs can be, for example, optimized for the overall TAU and paging overhead, that is, the corresponding $\boldsymbol{S}$-matrix yields the minimum sum of (2) and (3).

We apply a min-max approach for congestion mitigation, that is, TALs are used to minimize the maximum TAU between all pairs of TAs. To this end, an upper bound on the increase of the total paging overhead is necessary, as otherwise the optimum would be to completely eliminate TAU by exclusively using the TAL containing all TAs (i.e., as if all sites form one TA). The bound is expressed as $\beta C^{P}$, where $C^{P}$ is the total paging overhead of TA design $\mathcal{T}$, and $\beta$ is a non-negative parameter denoting the allowed percentage of increase. 
The set of all possible TALs of $\mathcal{T}$ is denoted by $\mathcal{L}$. Thus $\mathcal{L}$ is the set of all non-empty subsets of $\mathcal{T}$. We use $u_{v}$ to denote the total load parameter of all sites inside TA $v \in \mathcal{T}$, and $h_{v w}$ the total handover between TAs $v$ and $w$. These values are easily derived from the load and handover values between sites. Parameter $a_{v \ell}$ is a binary indicator, denoting whether or not TA $v$ is in TAL $\ell$. Moreover, $n_{v}$ denotes the number of sites in TA $v$. The aforementioned continuous variable $x_{\ell}$ represents the proportional use of TAL $\ell$ by all sites of the TAs in $\ell$. We use an auxiliary variable $z$ to denote the maximum TAU between TAs. The min-max TAU optimization problem can then be modeled by the following linear program (LP).

$\min z$

s. t. $\sum_{\ell \in \mathcal{L}} a_{v \ell} x_{\ell}=1, \quad v \in \mathcal{T}$

$$
c^{u}\left(h_{v w} \sum_{\ell \in \mathcal{L}} a_{v \ell}\left(1-a_{w \ell}\right) x_{\ell}+h_{w v} \sum_{\ell \in \mathcal{L}} a_{w \ell}\left(1-a_{v \ell}\right) x_{\ell}\right) \leq z, \quad v, w \in \mathcal{T}, v \neq w
$$

$$
\begin{aligned}
& \alpha c^{p} \sum_{\ell \in \mathcal{L}} \sum_{v \in \ell} \sum_{w \in \ell, v \neq w} u_{v} n_{w} x_{\ell} \leq \beta C^{P}, \\
& x_{\ell} \geq 0, \quad \ell \in \mathcal{L} .
\end{aligned}
$$

The first constraint set (7b) assures that, within any TA, the proportional usage values of TALs containing the TA sum up to one. This is the generalization of (4)-(6). The next set of constraints (7c) defines the maximum TAU between TA pairs. For each ordered pair $v$ and $w$, there is a contribution of $c^{u} h_{v w} x_{\ell}$, if TAL $\ell$ contains $v$ (i.e., $a_{v \ell}=1$ ) but not $w$ (i.e., $a_{w \ell}=0$ ). Constraints $(7 \mathrm{~d})$ bound the overall paging overhead. For any list $\ell$, the overhead 


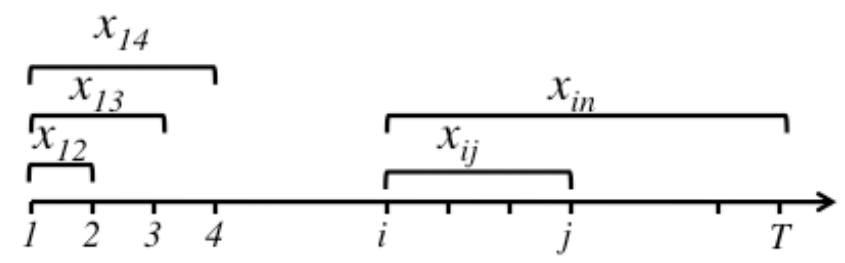

Figure 2: Definition of variables for the train scenario.

imposed by paging UEs in TA $v \in \ell$ to TA $w \in \ell$ is $\alpha c^{p} u_{v}$, scaled by the number of sites in $w$ and variable $x_{\ell}$ that stands for the proportional use of $\ell$.

At the optimum of (7), the $x$-variables are typically fractional. As a result, some but not all of the UEs moving from one TA to another generate TAUs, in contrast to the original TA layout. In other words, the sharp boundaries between the TAs are "blurred" by the fractional use of TALs.

The number of variables in (7) is exponential in the number of TAs $T$. However, in practice this is not problematic for two reasons. First, the region with high risk of excessive TAU is typically confined to a part of the network. Second, as (7) is an LP, it can be solved efficiently even for a large number of variables.

The LP formulation (7) applies to TAs of general topology. For the train scenario, the formulation takes a simpler structure, as the TAs involved in the scenario form a line. UE movement occurs only between consecutive TAs along the line. Thus, the TALs contain blocks of consecutive sites, and the formulation's size becomes polynomial in the number of TAs, see Figure 2. As numbering the TAs follows naturally their positions along the line, for convenience, we use the position indices to refer to TAs. Hence, TA $i$ is the 
$i$ th TA on the line, without the calligraphic style in the notation that has hitherto been used for sets. Each TAL is uniquely identified by its starting and ending TAs, therefore the variable definition is simplified to $x_{i j}$ for the TAL containing $i, j$ and all the TAs between them. The corresponding LP formulation is given below.

$$
\begin{array}{ll}
\min & z \\
\text { s. t. } & \sum_{i=1}^{k} \sum_{j=k}^{T} x_{i j}=1, \quad k=1, \ldots, T \\
& c^{u}\left(h_{i, i+1} \sum_{k=1}^{i} x_{k i}+h_{i+1, i} \sum_{k=i+1}^{T} x_{i+1, k}\right) \leq z, \quad i=1, \ldots, T-1 \\
& \alpha c^{p} \sum_{i=1}^{T-1} \sum_{j=i+1}^{T} \sum_{k=i}^{j} \sum_{h=k+1}^{j}\left(u_{k} n_{h}+u_{h} n_{k}\right) x_{i j} \leq \beta C^{P}, \\
& x_{i j} \geq 0, \quad i=1, \ldots, T, j=i, \ldots, T .
\end{array}
$$

Constraints (8b)-(8d) are respectively obtained by adapting (7b)-(7d). For the train scenario, it is sufficient to account for UE movements between adjacent TAs on the line, as can be seen in (8c). In (8d), paging is considered for all TA pairs of a line segment, and further over all the segments. Apart from the non-negativity requirement, the numbers of variables and constraints in (7a) are of $O\left(T^{2}\right)$ and $O(T)$, respectively. Thus, the optimization approach has very low complexity in the train scenario.

Consider two consecutive TAs, $i$ and $i+1$. It is easily verified that there are a total of $i(T-i+1)$ TALs containing $i$, and, among them, $i$ TALs do not contain $i+1$. Thus, the amount of TAU generated by UE movement from $i$ to $i+1$ depends on the proportional usage levels of the $i$ TALs. Typically, 
the values are strictly between zero and one at optimum, thus not all of the UEs inside the train will perform TAU at a TA boundary, giving the desired effect of TAU congestion mitigation.

\section{TAL Optimization for Congestion Mitigation of Paging}

The min-max optimization approach is not limited to congestion mitigation of TAU. It is easily adapted to reducing the risk of signaling congestion in paging that may become of concern for scenarios where massive UEs are populated densely in some area with little mobility. The paging overhead in this area becomes considerably higher than that in the rest of the network. To reduce the paging overhead, some type of area splitting needs to take place. However, if the area would be in one common TA, such a split would not be possible without reconfiguring the TAs into smaller ones. For clarity in our discussion, we assume that every single site is a TA in the current configuration. The assumption does not impose excessive TAU, because the effect of "TA" of any size equivalently can be achieved by using a TAL containing the corresponding single-site TAs $[8,17]$. Thus, in this section, it is assumed that non-overlapping TALs contain single-sited TAs. Each TAL has the function of one TA a conventional sense. For mitigating paging congestion, new (and smaller) TALs are created within the original ones, and their proportional usage levels are determined by min-max optimization.

Let $\mathcal{T}=\{1, \ldots, T\}$ be the set of single-site TAs in the TAL containing the area with risk of paging congestion. As in the previous section, $\mathcal{L}$ is used to denote the non-empty collections of elements of $\mathcal{T}$. The other variable and parameter definitions are reused as well. Note that within $\mathcal{T}$, TAU is zero for 
the mentioned configuration (single TAL containing all TAs of $\mathcal{T}$ ). Reducing paging would lead to a positive amount of TAU, and the limit is denoted by $C_{\text {lim }}^{U}$. The min-max LP formulation of paging congestion mitigation is provided below.

$\min z$

s. t. $\sum_{\ell \in \mathcal{L}} a_{v \ell} x_{\ell}=1, \quad v \in \mathcal{T}$

$\alpha c^{p} \sum_{v \in \mathcal{T}} u_{v} \sum_{\ell \in \mathcal{L}} a_{v \ell} a_{w \ell} x_{\ell} \leq z, \quad w \in \mathcal{T}$

$$
\sum_{v \in \mathcal{T}} \sum_{w \in \mathcal{T}: v \neq w} c^{u}\left(h_{v w} \sum_{\ell \in \mathcal{L}} a_{v \ell}\left(1-a_{w \ell}\right) x_{\ell}+h_{w v} \sum_{\ell \in \mathcal{L}} a_{w \ell}\left(1-a_{v \ell}\right) x_{\ell}\right) \leq C_{\lim }^{U},
$$

$$
x_{\ell} \geq 0, \quad \ell \in \mathcal{L}
$$

The first set of constraints are identical to that of (7). Constraints (9c) define, together with the objective, the maximum paging. For each singlesite TA $\boldsymbol{w}$, the left-hand side of (9c) represents the total amount of overhead generated to $w$ because of paging UEs located in TAs other than $w$, due to the use of TALs with multiple sites. The next constraint set is the aggregated form of $(7 \mathrm{c})$ to represent the total TAU, bounded by the limit value in the right-hand side.

The limit value in (9) and parameter $\beta$ in (7) are not necessarily considered constants prior to running TAL optimization. In fact, the LP can be solved repeatedly for a range of limit values. Doing so generates a set of candidate solutions representing various levels of the trade-off between congestion reduction of TAU and the price of more paging, and vice versa (cf. 
Pareto optimization).

In previous studies of applying TALs, e.g., $[8,16]$, the performance comparison relies heavily on the assumptions made on UE mobility and other network parameters, because in general, UEs that are initially registered to the same TAL by a site will spread out within the area defined by TAL. After some time duration, the proportion of UEs belonging to the TAL will become heterogeneous over the area, as a result of each UE's individual movement. Note that the amount of TAU and paging generated by a UE are both dependent on the content of its TAL. Therefore, unless a very accurate mobility model is available (which is hardly the case in many scenarios), the practical performance of TAL may deviate significantly from the estimated one.

In our overlapping TAL scheme, the construction of the LPs ensures the same proportional use of each TAL by all sites within the TAL. Therefore, statistically the proportion of UEs of each TAL remains homogeneous with the TAL's area, meaning that the performance is insensitive to individual UE movement. Provided that the location distribution and mobility pattern of idle UEs are similar to those of active UEs, which is a well-accepted assumption, the performance of the overlapping TAL scheme is conveniently assessed. This makes the performance comparison both simple and unbiased, and its validity is not impacted by mobility models.

\section{Performance Evaluation}

In this section we present results of performance evaluation of the proposed TAL optimization framework. In Section 6.1, we provide performance evaluation of TAU congestion mitigation for a train scenario. Next, the re- 
sults of reducing TAU as well as paging congestion for a general network topology are reported in Section 6.2.

In the performance evaluation, the overhead of a single TAU, $c^{u}$, is set ten times as much as $c^{p}[12]$. Parameter $\alpha$ is set to 0.05 , i.e. $5 \%$ of the UEs are paged. For all the scenarios, there is an underlying TA layout (possibly implemented via TALs) that represents the most likely design in terms of planning. Namely, the TA layout is the global optimum in minimizing the overall signaling overhead (i.e., $C^{P}+C^{U}$ ). This optimum TA layout is computed using the integer programming formulation in [27] and software CPLEX [11].

Whereas comparing the performance of the proposed TAL scheme with the underlying TA design gives insights, it is somewhat biased to the favor of the former, because the latter is not optimized for signaling congestion but for the overall signaling overhead. For this reason, the performance comparison is conduced between overlapping TALs and a re-optimization of TA layout for the objective metrics of this paper, that is, the TAs can be merged for the objective function (7a) or split for the objective function (9a). This gives an unbiased assessment of optimized overlapping TALs.

The trade-off between the signaling overhead of TAU and paging has to be dealt with in both the conventional TA scheme and the proposed overlapping TAL scheme. For the latter scheme, reducing TAU congestion by using overlapping TALs results in overall higher paging in comparison to the baseline TA design, and vice versa. This aspect is reflected by the limit parameters in (7), (8), and (9). For the limit parameter $\beta$ in (7) and (8), the minimum possible value is zero. In this case, no additional paging is permit- 
ted, and consequently the only feasible solution is to reuse the baseline TA design. That is, the conventional TA scheme and overlapping TAL scheme coincide. When $\beta$ increases, the concern of paging overhead is diminishing, and both schemes will eventually coincide again, giving the same solution with one single TA (or TAL) containing all sites with zero TAU overhead. At this point, constraint (7d) is void, and increasing $\beta$ further clearly has no effect. A similar observation applies to the TAU limit in (9). Namely, the two schemes coincide when the limit is zero for which the baseline TA design is kept, or when the limit is very large such that the optimum for both is to split TAs maximally with zero paging (but completely sacrifices TAU). Thus, for each of the limit parameters, there is a maximum value of significance.

In our performance evaluation, for each limit parameter we first find its maximum value of significance, using bi-section search for the given planning scenario. Then, within the range of significance, we conduct computations for 500 equally-spaced values of the limit parameter, in order to give a comprehensive performance picture. The results represent, in fact, a large number of possible options for the trade-off between TAU and paging, both for the conventional TA scheme and for the overlapping TAL scheme, allowing the network operator to select the solution to implement, based on the operator's preference.

\subsection{Results for a Train Scenario}

To evaluate the performance of the proposed overlapping TAL scheme for the train scenario, a railway path with five TAs $\{A, B, C, D, E\}$ of 150 $\mathrm{km}$ in length is considered (see Figure 3). Each TA contains five sites. The configuration is the optimum conventional TA design with minimum total 


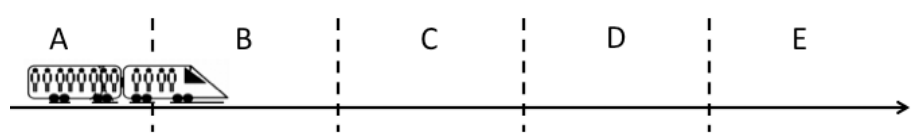

Figure 3: The train scenario.

signaling overhead, $C^{U}+C^{P}$, by solving the model in [27], for a baseline scenario that does not account for the massive mobility of UEs due to train movement. For this TA design, the total signaling overhead is 841.7, with $C^{U}=122$ and $C^{P}=719.7$.

In the train scenario (Figure 3), a train moves from $A$ to $E$ having 600 passengers on-board. One example can be TGV Eurostar train which can move at a speed up to $300 \mathrm{~km} / \mathrm{h}$ and may carry up to 784 passengers [14]. After a main station in $C$, the number of on-board passengers is reduced to 400. We assume that all passengers inside the train hold cell phones belonging to the same operator. It takes about half an hour for the train to pass the whole path (ignoring the time spent at the main station).

We aggregate the load and handover data resulted from the train movement and add them to the baseline scenario. All the UEs in the train passing each TA boundary add to the corresponding handover. The load is increased accordingly, taking into account the proportion of time the train is spent in each TA. For example, the train spends about 6 minutes in $A$, therefore the load of $A$ is increased by $600 \times \frac{1}{10}=60$, due to the fact that the handover and load data should be specified for one hour period of time.

We apply LP formulation (8) to the train scenario for $\beta \in[0,0.8]$, which is the range of significance for the scenario. Next, for the same range of $\beta$, we re-design the TAs by minimizing the maximum TAU using objective 


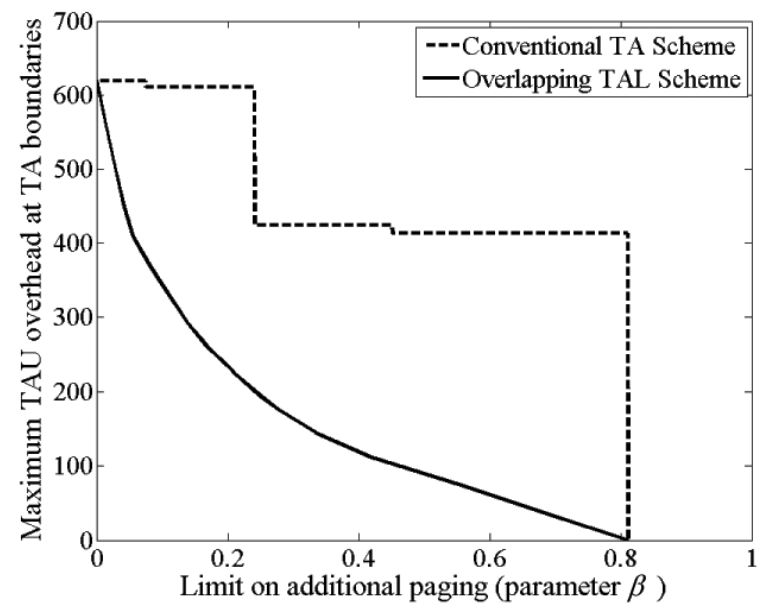

Figure 4: A performance comparison for the train scenario.

(8a). For $\beta=0$ and $\beta \geq 0.8$, the two schemes produce the baseline TA design (with non-overlapping TALs for the TAL scheme) and the all-in-one TA (or TAL) design, respectively. For the other $\beta$ values, Figure 4 illustrates the behavior of the two schemes. The horizontal axis is the upper limit on additional paging (i.e., parameter $\beta$ ), the vertical axis is the maximum TAU between TAs. The values are shown respectively by solid and dashed lines for optimized layout of overlapping TALs and that of the conventional TA scheme.

It is observable that the conventional TA scheme is changed in four discrete steps. The behavior of the overlapping TAL scheme is smooth, as the proportional use of each TAL can be continuously changed. Taking $\beta=0.2$ as an example, with the conventional TA scheme the maximum TAU is reduced by $1.45 \%$ only, while the overlapping TAL scheme reduces the maximum TAU by $62.3 \%$. This case study demonstrates the benefit of the overlapping TAL scheme in mitigating TAU due to massive mobility in the train scenario. 


\subsection{Performance Assessment in a Large-scale Network}

For performance evaluation on a general and large-scale topology, we use a realistic cellular network scenario for the downtown area of Lisbon. The scenario is provided by the MOMENTUM EU project [19]. The network consists of 60 sites and 164 cells. Figure 5(a) illustrates the network topology. The sites are represented by disks. For every site, its cells are denoted by squares. The location of a square in relation to the site center shows the direction of cell antenna. The darkness of each cell is set in proportion to its accumulated cell load. A link is drawn between two cells if there is any handover between them, and the amount of handover is proportional to the thickness of the link.

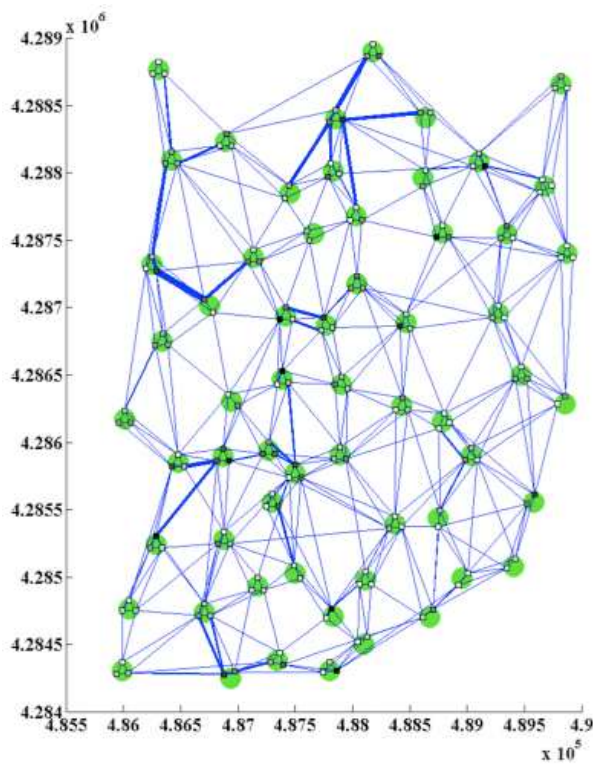

(a)

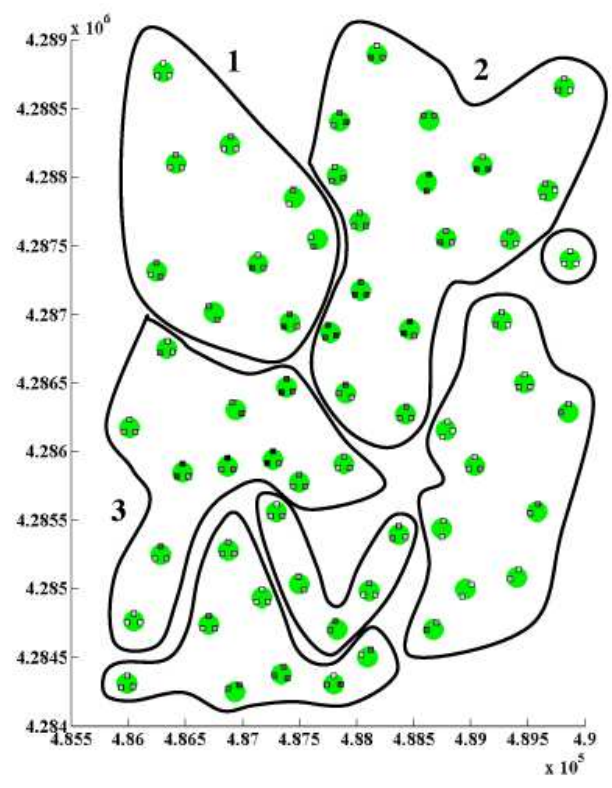

(b)

Figure 5: An illustration of (a) Lisbon network topology and key statistics, (b) the underlying TA design. 


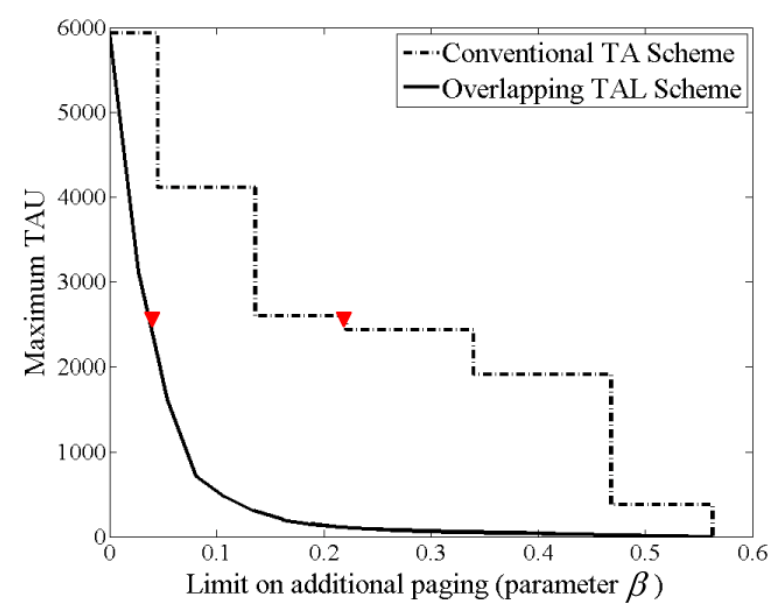

Figure 6: Performance evaluation of TAU congestion mitigation.

Figure 5(b) illustrates the underlying TA layout (i.e., the optimum with respect to the overall signaling overhead). There are seven TAs in the design; these are used for creating overlapping TALs. Note that, in mitigating signaling congestion of paging, the functionality of the conventional TA scheme is implemented with non-overlapping TALs of single-sited TAs (see Section 5). In this case, we will use the term TA group for the sake of clarity. Key statistics of the TA design are as follows. The overall TAU overhead $C^{U}=23849$, the overall paging overhead $C^{P}=18603$. The highest TAU between TAs, denoted by $C_{\max }^{U}$, equals 5996, and the most congested site in paging has a paging overhead of $C_{\max }^{P}=1058$.

For parameter $\beta$, the range of significance in this case is $[0,0.56]$. Formulation (7) in Section 4 is solved with $\beta$ varying within the range. The same range is applied in re-optimizing TAs with the conventional TA scheme for the same objective function (7a). The results are displayed in Figure 6 and Table 2. In addition to signaling overhead, the table contains information of 
Table 2: Performance comparison and computing time for TAU congestion mitigation.

\begin{tabular}{ccccc}
\hline$\beta$ & \multicolumn{2}{c}{ Conventional TA scheme } & \multicolumn{2}{c}{ Overlapping TAL scheme } \\
& Maximum TAU & Time (ms) & Maximum TAU & Time (ms) \\
0.0 & 5963 & - & 5953 & 8 \\
0.1 & 4109 & 52 & 537 & 12 \\
0.2 & 2953 & 52 & 131 & 12 \\
0.3 & 2423 & 76 & 62 & 12 \\
0.4 & 1910 & 60 & 37 & 12 \\
0.5 & 373 & 230 & 14 & 12 \\
\hline
\end{tabular}

the computing time.

As was explained in the case of train scenario, the performance curves coincide at the two ends. One immediately observes that the curve of the overlapping TAL scheme is smooth, whereas that of the conventional TA scheme has a stepwise shape. For the latter, the TAs may not overlap due to the binary and transitive properties of the $\boldsymbol{S}$ matrix (Section 3). As a result, the only possibility of reducing the maximum TAU is by merging TAs. This inherent limitation of the conventional TA scheme leads to the stepwise shape. Overlapping TALs, in contrast, can achieve "partial merge" of TAs, by solving the optimization formulation (7), yielding a smooth curve that significantly outperforms the conventional TA scheme.

For example, if the overall paging overhead is allowed to increase by $10 \%$, re-optimizing TAs by the conventional TA scheme reduces the peak TAU between TAs from $C_{\max }^{U}=5996$ to slightly above 4109. Using optimized overlapping TALs, the peak is pushed down to 537, see Table 2. This is 
only one seventh of the maximum TAU resulted from the conventional TA scheme. Thus TAU signaling congestion can be effectively handled by the proposed TAL scheme with little price paid for a higher amount of paging.

Examining the computing time in Table 2, one immediately observes that solving (7) for TAU mitigation runs very fast, and the computing time is not sensitive at all to the limit value. In fact, the overlapping TAL scheme runs several times faster than the conventional TA scheme. In conclusion, the former has superior performance both in TAU mitigation and the computational burden.

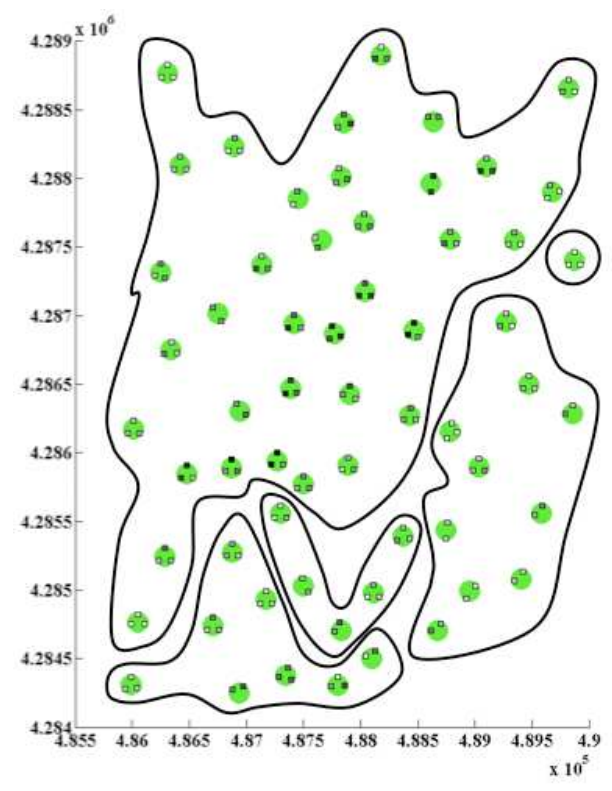

(a)

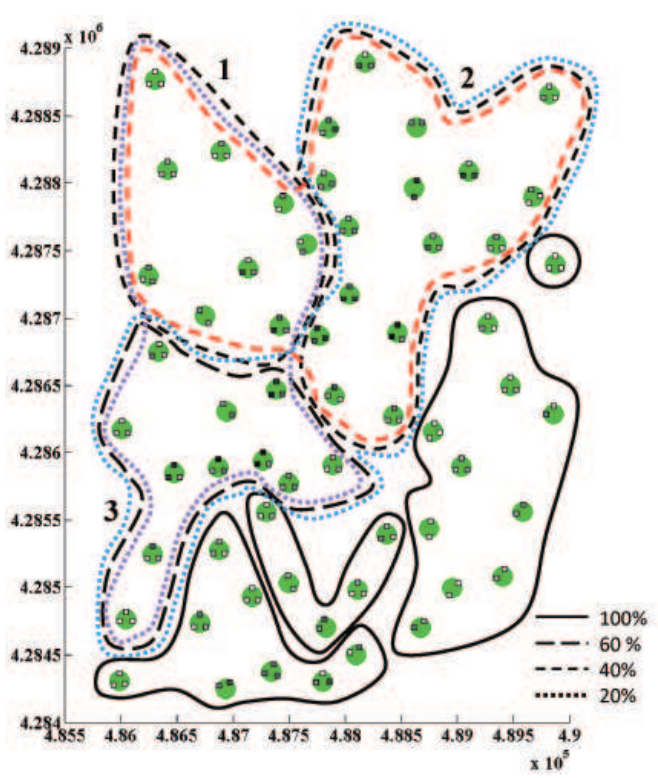

(b)

Figure 7: An illustration of the design by (a) the conventional TA scheme and (b) overlapping TAL, for selected value of maximum TAU.

Figure 7 provides an illustration of the designs corresponding to the two markers in Figure 6. The conventional TA scheme and overlapping TAL 


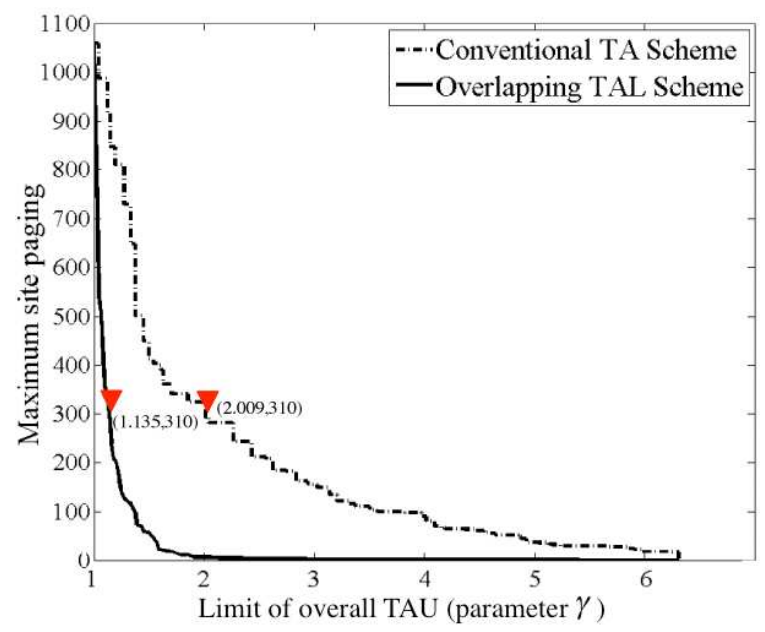

Figure 8: Performance evaluation of paging congestion mitigation.

give the same level of peak TAU (approximately 2400) for selected solutions, although the former has considerably higher paging. Figure 7(a) shows the TA layout given by the conventional TA scheme; one can see this layout is a simple merge of TAs 1-3 in Figure 5(b). The composition and usage of TALs are shown in Figure 7(b). The original three TAs are still used, but to lesser extent. In addition, TALs containing pairs of TAs are in place, with the effect of reducing TAU peak between them. The illustration highlights the effect of blurring TA boundaries by overlapping TALs.

As the last part of our performance evaluation, we apply the overlapping TAL scheme for mitigating paging congestion, using Figure 5(b) as the base layout. In this case, the TAs are assumed to be single-sited, and the grouping in Figure 5(b) is implemented by means of non-overlapping TALs (see Section 5). Overlapping TALs are then created and optimized by formulation (9) to achieve partial split within each TA group. The TA groups are also reoptimized following the conventional principle of TA design; this corresponds 
Table 3: Performance comparison and computing time for paging congestion mitigation.

\begin{tabular}{ccccc}
\hline$\gamma$ & Conventional TA scheme & \multicolumn{2}{c}{ Overlapping TAL scheme } \\
& Maximum paging & Time $(\mathrm{s})$ & Maximum paging & Time (s) \\
1.0 & 1058 & - & 1058 & $<1$ \\
1.2 & 847 & 45 & 201 & 33 \\
1.4 & 501 & 555 & 72 & 94 \\
1.6 & 400 & 340 & 22 & 1881 \\
1.8 & 340 & 517 & 11 & 259 \\
2.0 & 324 & 378 & 6 & 384 \\
\hline
\end{tabular}

to finding the optimal split (with sharp boundaries) for objective function (9a). The performance comparison is given in Figure 8 and Table 3. For the sake of compactness, the results represent the aggregated values over all TA groups. Thus $C_{\lim }^{U}$ can be set in relation to $C^{U}$ of the baseline design. We denote by $\gamma$ the ratio between $C_{\lim }^{U}$ and $C^{U}$. The range of significance for the scenario is $\gamma \in[1.0,6.3]$.

In the figure, the horizontal axis is $\gamma$, For example, $\gamma=2.0$ on the axis means that the $C_{\lim }^{U}=2 C^{U}$. The vertical axis represents the maximum paging overhead generated by the sites. For each site, this overhead is the amount of paging overhead sent by the site in order to locate UEs of other sites. When $\gamma=1.0$, the only feasible solution is to keep the baseline TA groups, for which the maximum site paging equals $C_{\max }^{P}$. The maximum decreases gradually in respect of $\gamma$, and reaches zero (i.e., full split) when $\gamma \geq 6.3$. Note that in Table $3, \gamma$ is limited to 2.0, because the maximum paging in the overlapping TAL scheme is virtually zero beyond this point. 
In comparison to Figure 6, the performance curve of the conventional TA scheme in Figure 8 is much smoother. This is because splitting a TA group can be done with a much better granularity (e.g., one site at a time) than merging TAs. Nevertheless, the performance is evidently inferior to that of adopting overlapping TALs, as can be seen from Figure 8 and Table 3. The overlapping TAL scheme is able to reduce the maximum paging much more steeply in comparison to conventional split of TA groups. For example, the former virtually eliminates paging, if the TAU is allowed to be doubled (i.e., $\gamma=2.0$ ), whereas in the latter case the maximum paging is still above one third of the baseline value, as shown in Table 3. Moreover, the magnitude of the performance difference grows in $\gamma$ for the range considered in the table. As another comparison, recall that the average site paging of the baseline design is approximately 310 (that is, $C^{P}$ divided by the number of sites), and suppose we would like to bring the maximum paging level down to this value, for which the two markers in the figure show the performance of the two schemes. Using the conventional TA scheme (or equivalently nonoverlapping TALs), the TAU overhead has to be doubled. With optimized overlapping TALs, the effect is achieved with merely a $13 \%$ increase in TAU. Thus overlapping TALs provide an effective means of splitting hotspots for avoiding paging congestion.

In Table 3, the computing time used by the overlapping TAL scheme is up to 30 minutes. The major part of the time is attributed to the largest TA group with 16 sites, see Figure 5(b). For this TA group, the LP (9) contains $2^{16}-1$ variables. Although the time range may not admit on-line use, it remains reasonable in a planning stage, because typically a hotspot 
of massive and close-to-static UEs can be predicted in advance. We observe also that time values for the overlapping TAL scheme and the conventional TA scheme are comparable in magnitude, and the former scheme runs faster for all except one of the cases in the table.

\section{Conclusions}

We have proposed a scheme using overlapping tracking area lists (TALs) and a min-max optimization approach for the purpose of mitigating signaling congestion for location management in LTE networks. The optimization task was formulated and computed by means of linear programming. As the key aspect of the optimization framework, consistency in the proportional use of TALs is maintained throughout the network, and consequently no assumption or model of UE mobility is required in order to assess the performance of overlapping TALs. Numerical evaluations of mitigating massive TAU as well as paging congestion demonstrate that the proposed optimization framework is very effective. Optimization of overlapping TALs significantly outperforms the conventional TA scheme in signaling congestion. By providing high gran-

ularity in re-distributing the signaling overhead over the network area, TALs open up a new dimension of freedom in location management.

An interesting line of extension of the current work is dynamic configuration of overlapping TALs. By monitoring TAU and paging statistics and adapting the proportional use of TALs accordingly, the proposed TAL scheme can be extended to form an automated process in location management within the paradigm of self-optimization networks. This topic will be addressed in our forthcoming research. 


\section{Acknowledgments}

The work has been supported by CENIIT centrum of Linköping University, the Swedish Research Council, and the Swedish Foundation of Strategic Research.

\section{References}

[1] 3GPP. TS 23.002 - v9.1.0, Network Architecture, 3G Release 9, 2009. http://www.3gpp.org/ftp/specs/archive/23_series/23.002/.

[2] 3GPP. TS 23.401 - v8.6.0, General Packet Radio Services (GPRS) enhancements for Evolved Universal Terrestrial Radio Access Network (EUTRAN) access, 3G Release 8, 2009.

http://www.3gpp.org/ftp/specs/archive/23_series/23.401/.

[3] 3GPP. TR 23.880 - v0.1.0, Stage2 for registration in densely-populated area (RED), 3G Release 8, 2007.

http://www.3gpp.org/ftp/specs/archive/23_series/23.880/.

[4] I.F. Akyildiz, J.S.M. Ho, Y.B. Lin, Movement-based location update and selective paging for PCS networks, ACM/IEEE Transactions on Networking 4 (1996) 629-638.

[5] I.F. Akyildiz, J. McNair, J.S.M. Ho, H. Uzunalioglu, W. Wang, Mobility management in next generation wireless systems, Proceeding of IEEE 87 (1999) 1347-1384. 
[6] Y. Bejerano, M.A. Smith, J. Naor, N. Immorlica, Efficient location area planning for personal communication systems, ACM/IEEE Transaction on Networking 14 (2006) 438-450.

[7] E. Cayirci, I.F. Akyildiz, Optimal location area design to minimize registration signaling traffic in wireless systems, IEEE Transactions on Mobile Computing 2 (2003) 76-85.

[8] Y.W. Chung, Adaptive design of tracking area list in LTE, in: Proceeding of 8th IEEE International Conference on Wireless and Optical Communications Networks (WOCN), Paris, 2011.

[9] I. Demirkol, C. Ersoy, M.U. Caglayan, H. Delic, Location area planning and cell-to-switch assignment in cellular networks, IEEE Transactions on Wireless Communications 3 (2004) 880-890.

[10] P. Garcia, V. Casares, J. Mataix, Reducing location update and paging costs in a PCS network, IEEE Transactions on Wireless Communications 1 (2002) 200-209.

[11] ILOG CPLEX 12.2, User's Manual, 2010.

[12] K. Kyamakya, K. Jobmann, Location management in cellular networks: classification of the most important paradigms, realistic simulation framework, and relative performance analysis, IEEE Transactions on Vehicular Technology 54 (2005) 687-708.

[13] T. Mach, R. Tafazolli, Mass mobility signaling congestion avoidance mechanism using randomized time distribution of cell reselections, in: 
Proceedings of International Conference on Telecommunications (ICT), Marrakesh, 2009, pp. 238-242.

[14] Mitsubishi Electric, Tracking areas sizes and tracking area list optimization, Technical Report in 3GPP TSG RAN WG3 Meeting, R3-071931, 2007.

[15] S. Modarres Razavi, D. Yuan, F. Gunnarsson, J. Moe, Performance and cost trade-off in tracking area reconfiguration: a Pareto-optimization approach, Journal of Computer Networks 56 (2012) 157-168.

[16] S. Modarres Razavi, D. Yuan, F. Gunnarsson, J. Moe, Exploiting tracking area list for improving signaling overhead in LTE, in: Proceedings of IEEE Vehicular Technology Conference (VTC Spring), Taipei, 2010.

[17] S. Modarres Razavi, D. Yuan, F. Gunnarsson, J. Moe, Dynamic tracking area list configuration and performance evaluation in LTE, in: Proceedings of IEEE Global Communications Conference Workshop (GLOBECOM Workshop), Miami, 2010.

[18] S. Modarres Razavi, D. Yuan, Mitigating mobility signaling congestion in LTE by overlapping tracking area lists, in: Proceedings of the 14th ACM International Conference on Modeling, Analysis and Simulation of Wireless and Mobile Systems (MSWiM), Miami, 2011, pp. 285-292.

[19] IST-2000-28088 Momentum project. http://momentum.zib.de.

[20] Motorola, Long Term Evolution (LTE): A technical overview, Technical White Paper, 2007. 
[21] Z. Naor, H. Levy, Minimizing the wireless cost of tracking mobile users: an adaptive threshold scheme, in: Proceedings of IEEE Conference on Computer Communications (INFOCOM), San Francisco, 1998, pp. 720729 .

[22] G.P. Pollini, I. Chih-Lin, A profile-based location strategy and its performance, IEEE Journal on Selected Areas in Communications 15 (1997) 1415-1424.

[23] R. Prakash, Z. Haas, M. Singhal. Load-balanced location management for cellular mobile systems using quorums and dynamic hashing, Wireless Networks 7 (2001) 497-512.

[24] NEC, Tracking area concepts and influence on paging load, Technical Report in 3GPP TSG RAN2 Meeting, R2-070655, 2007.

[25] QUALCOMM Europe and T-Mobile, New SON user case: tracking area optimization, Technical Report in 3GPP TSG RAN WG3 Meeting, R3071594, 2007.

[26] S. Subramaniam, G. Krishnamurthi, Load balancing location management, in: Proceedings of IEEE International Conference on Communications (ICC), Helsinki, 2001, pp. 2835-2839.

[27] D.W. Tcha, T.J. Choi, Y.S. Myung, Location-area partition in a cellular radio network, Journal of the Operational Research Society 48 (1997) 1076-1081.

[28] T. Winter, Mobility management and network design for UMTS, in: 
Proceedings of IEEE International Symposium on Personal, Indoor and Mobile Radio Communications (PIMRC), 2004.

[29] W. Wang, I.F. Akyildiz, G.L. Stüber, B. Chung, Effective paging schemes with delay bound as QoS constraints in wireless systems, Wireless Networks 7 (2001) 455-466.

[30] V.W.S. Wong, V.C.M. Leung, Location management for next generation personal communication networks, IEEE Network 14 (2000) 18-24.

[31] Y. Zhang, M. Fujise, Location management congestion problem in wireless networks, IEEE Transactions on Vehicular Technology 56 (2007) 942-954.

[32] ZTE, Self optimization for tracking areas, Technical Report in 3GPP TSG RAN WG3 Meeting, R3-071415, 2007. 